\title{
Pathological issues in biopsy specimens of men with prostate cancer eligible for active surveillance
}

\author{
Roberta Mazzucchelli ${ }^{1}$, Andrea Benedetto Galosi ${ }^{2}$, Antonio Lopez-Beltran ${ }^{3}$, \\ Marina Scarpelli ${ }^{1}$, Liang Cheng ${ }^{4}$, Rodolfo Montironi ${ }^{1}$ \\ ${ }^{1}$ Section of Pathological Anatomy, Polytechnic University of the Marche Region, School of Medicine, United Hospitals, Ancona, Italy; \\ ${ }^{2}$ Division of Urology, "Augusto Murri" General Hospital, ASUR Marche, Fermo, Italy; \\ ${ }^{3}$ Department of Surgery, Cordoba University Medical School, Cordoba, Spain; \\ ${ }^{4}$ Department of Pathology and Laboratory Medicine, Indiana University School of Medicine, Indianapolis, IN, USA.
}

\begin{abstract}
Summary Active surveillance (AS) is an important management option for men with lowrisk, clinically localized prostate cancer. The clinical parameters for patient selection and definition of progression for AS protocols are evolving as data from several large cohorts become mature. Vital to this process is the critical role pathologic parameters play in identifying appropriate candidates for AS. These findings need to be reproducible and accurately reported by pathologists.

Repeated biopsy after initial diagnosis of prostate cancer is recommended before inclusion in active surveillance for early detection of significant cancer.
\end{abstract}

KEY WORDS: Prostate cancer; Prostate biopsy; Active surveillance; Tumour extent; Immunohistochemistry.

Submitted 3 October 2014; Accepted 31 October 2014

\section{INTRODUCTION}

The overwhelming majority of men diagnosed with prostate cancer $(\mathrm{PCa})$ opt for primary curative therapy, such surgery to remove the prostate, i.e., radical prostatectomy, or radiation therapy to eradicate the tumor. However, most PCas are indolent and the number of newly diagnosed cases far outnumbering that of lethal cases (1). The magnitude of this so-called overdiagnosis, where cancers are identified that would never progress or cause harm to the patient if left untreated, ranges from $15 \%$ to $84 \%$ of new PCa cases $(2,3)$. Patients are exposed to the risk of overtreatment. Even if overtreatment is avoided, overdiagnosis induces anxiety associated with the new cancer diagnosis, often resulting in further tests and expenses, and confers upon the patient a 'cancer survivor' label he carries for the rest of his life (4). Male offspring may worry about familial inheritance and increased risk of the disease.

Given the indolent course of many PCa detected by PSA screening (5), active surveillance (AS) has emerged as an initial management alternative, thus men avoiding the side effects of PCa treatment. Under most AS strategies, patients undergo careful monitoring of the cancer, most often with PSA kinetics and serial biopsy. Any progression of the cancer while patients are monitored appears unlikely to threaten length of life (6).

Through this report we aim at facilitating dissemination of information on the critical role pathological parameters play in identifying appropriate candidates for AS.

\section{Clinical perspective on AS}

Specific inclusion criteria for AS vary across institutions $(7,8)$. Patients are selected for AS on the basis of their age, PSA density (PSA/prostate volume), measures of PSA kinetics, such as PSA velocity, percent of positive biopsy cores, the extent of prostate cancer in any core, and Gleason score $3+3=6$ (9). Some of these cohorts include patients with intermediate-risk clinical parameters, allowing for inclusion of patients with PSA at diagnosis greater than $10 \mathrm{ng} / \mathrm{ml}$ or including selected men with Gleason $3+4=7$ PCa. Surveillance schedules for AS are variable across institutions. Most $\mathrm{PCa}$ experts agree that surveillance should include a combination of serial PSA and rectal examinations as well as repeat prostate biopsy. Biopsy grade reclassification has emerged as a more meaningful endpoint for men on AS. Higher-grade tumors (Gleason 7 and higher) clearly confer a higher likelihood of clinical progression. A finding of upgrading cancer on repeat biopsy commonly prompts treatment.

This upgrading may represent undersampling at the time of initial diagnostic biopsy where the pre-existing highgrade prostate cancer was missed (10). Tables 1 and 2 include, as an example, inclusion criteria, surveillance schedule and the definition of progression of three AS protocols.

\section{RepeATED BIOPSY}

Repeated biopsy after initial diagnosis of prostate cancer is recommended before inclusion in active surveillance, since repeated biopsy improves cancer grading and reduce the risk of undersampling of significant cancer. Repeated biopsy should follow criteria of saturation 
Table 1.

Active surveillance inclusion criteria for selected institutions (see text).

\begin{tabular}{|l|c|c|c|}
\hline Inclusion criteria & Johns Hopkins & University of Toronto & PRIAS \\
\hline $\mathrm{N}^{\circ}$ of patients & 870 & 453 & 2494 \\
\hline Clinical stage & $\mathrm{T} 1 \mathrm{c}$ & - & $\leq \mathrm{T} 2$ \\
\hline $\mathrm{PSA}$ density & $\leq 0.15 \mathrm{ng} / \mathrm{ml} / \mathrm{cc}$ & - & $\leq 0.20 \mathrm{ng} / \mathrm{ml} / \mathrm{cc}$ \\
\hline $\mathrm{PSA}$ & - & $\leq 15 \mathrm{ng} / \mathrm{ml}$ & $\leq 10 \mathrm{ng} / \mathrm{ml}$ \\
\hline No. of + ve cores & $\leq 2$ & & $\leq 2$ \\
\hline$\%$ cancer per core & $\leq \% 50$ & & - \\
\hline Gleason score & $\leq 6$ & $\leq 7(3+4)$ & $\leq 6$ \\
\hline
\end{tabular}

\section{Tumor extent measurements}

There is no consensus on the best tumor quantification methods, which include: cancer percentage in each core, greatest percentage of cancer, cancer length in each core, greatest length of cancer (GLC), total percentage of carcinoma in all cores, total length carcinoma in all cores, fraction of positive cores, total carcinoma surface area and total percentage of carcinoma surface area in all cores. Tumor measurements are performed as a visual estimate or using an ocular micrometer or other morphometric

Table 2.

Surveillance schedule and definition of progression for selected institutions (see text).

\begin{tabular}{|c|c|c|c|}
\hline \multirow[b]{2}{*}{ Institutions } & \multicolumn{2}{|c|}{ Surveillance schedule } & \multirow[b]{2}{*}{ Definition of progression } \\
\hline & PSA and DRE & Repeat prostate biopsy & \\
\hline Johns Hopkins & Every 6 months & Yearly & $\begin{array}{c}\text { Gleason score }>6 \text {, or }>2 \text { cores, } \\
\text { or }>50 \% \text { any core }\end{array}$ \\
\hline University of Toronto & $\begin{array}{l}\text { Every } 3 \text { months for } 2 \text { yrs, } \\
\text { then every } 6 \text { months }\end{array}$ & $\begin{array}{l}\text { 6-12 months after diagnosis, } \\
\text { then every 3-4 years }\end{array}$ & PSADT $<3$ years \\
\hline PRIAS & $\begin{array}{l}\text { Every } 3 \text { months for } 2 \text { yrs } \\
\text { and then every } 6 \text { months }\end{array}$ & $\begin{array}{l}1,4,7 \text { and } 10 \text { yrs } \\
\text { after diagnosis }\end{array}$ & $\begin{array}{c}\text { GS }>6, \text { or } \geq 3 \text { positive cores, } \\
\text { or PSADT }<3 \text { yearly }\end{array}$ \\
\hline
\end{tabular}

biopsy (20 core or more based on prostate volume) (11). Anterior gland should be included in the repeated biopsy. Magnetic Resonance imaging can be performed before re-biopsy since the negative predictive value for significant cancer raise 95\%, however the positive predictive value is lower. Men who experience early upgrading likely represent initial sampling error, whereas later upgrading may reflect tumor dedifferentiation.

\section{The role of the Pathologist}

The essential reporting items for cancer containing prostatic needle biopsies are listed in Table 3. Tumor extent measurements and the Gleason score are the most important pathologic parameters in needle biopsies determining eligibility for AS protocols.

Table 3.

Essential reporting elements for cancer bearing prostatic needle biopsies.

\begin{tabular}{l} 
1. Location of positive cores \\
\hline 2. Tumor extent \\
\hline 3. Gleason grades and score \\
\hline 4. Histologic type \\
\hline 5. Other (reported only if present) \\
- Extraprostatic extension \\
- Perineural invasion \\
- Lymphovascular invasion \\
- Intraductal carcinoma \\
6. Other atypical acinar foci suspicious for carcinoma
\end{tabular}

measurement such as computerized methods. Visual estimation of percentage without morphometric measurements is commonly performed, although many recent studies do not actually describe whether visual estimation or morphometric measurements were used. Some use a regular ruler or the side graticule available on most microscopes for estimation of length and percentage. The knowledge of the diameter of the field at each magnification for the microscope used to measure tumor extent can also help maximize accuracy of visual estimation of length. In a recent abstract, Mahamud et al. found no overall difference between visual estimation and measurement when determining percent involvement of prostate biopsies assessed only by whole slide images. However, there was a significant difference between the two methods when they considered a subset of cores deemed to have $40-60 \%$ involvement by visual estimation. It is unclear whether the accuracy of visual estimation of an image can be compared with that of a tissue core on a glass slide on a microscope. Data are conflicting whether morphometric measurements are superior to visual estimation and whether differences in the two methods would affect clinical management.

Computerized morphometric measurements are considered time-consuming and not practical for most pathologists. Measurements of core length given in gross descriptions should not be used as these may not always be accurate.

A few studies have assessed the value of the different methods of tumor extent measurement in prostate needle biopsy in predicting pathological stage or prognosis. Quintal et al. (12) found that total percentage of carcinoma in all cores and number and percentage of cores with cancer were significantly stronger than other methods 
such as greatest percentage of cancer or length in a single core in predicting biochemical recurrence. Total percentage of carcinoma in all cores had the strongest correlation and when combined with preoperative PSA and Gleason score improved prediction of pT3 in multivariate analysis. This was also independent for risk of biochemical recurrence. Bismar et al. (13) found that although many tumor measurements such as greatest percentage of cancer, total tumor length in millimeters, fraction of positive cores and total percentage of carcinoma were significant in univariate analysis, only the fraction of positive cores was significant in multivariate analysis in predicting pT3 disease or positive margins. In this study all the measures were highly related to one another in a formal correlation analysis.

Park et al. (14) examined the significance of the number of cores positive for cancer, percentage of positive biopsy cores, total linear cancer length, total percentage of carcinoma and maximum cancer core length and found that, when considering PSA and Gleason score, none were significant in predicting pT3 disease in multivariate analysis. In a study by Brimo et al. (15), it was found that the fraction of positive cores, total percentage of carcinoma and both total and greatest cancer core length were closely associated with pathological stage and biochemical failure. The fraction of positive cores was found to be the factor most closely associated with pT3 disease in radical prostatectomy.

Correlating needle biopsy cancer measurements with tumor volume in radical prostatectomy, Poulos et al. (16) found that the highest percentage of carcinoma in any biopsy site, percentage of adenocarcinoma at the biopsy site with the highest grading, the number of positive biopsy sites and tumor bilaterality were significant with the percentage of biopsy sites positive for disease the most significant predictor of tumor volume. In a study by Lewis et al. (17), tumor volume was best predicted by a combination of linear extent of carcinoma and number of positive cores.

In a survey sent to 93 genitourinary pathologists the extent of cancer on needle biopsies was quantified by all the respondents with $80 \%$ reporting the number of cores involved by cancer. Linear extent was estimated by almost all, either as a percentage (80\%) or millimeters of cancer length (41\%) or both (22\%).

Considering the tumor quantification methods actually requested by urologists, in a 2005 study, 95\% French and Belgian urologists requested the number of positive cores compared with 53\% requesting length of cancer. In a study by Rubin et al. (18) $3067 \%$ of urologists requested the percent involvement of each core by cancer, $33 \%$, the number of cores with prostate cancer and 29\% the length of core involvement.

Recommendations by the College of American Pathologists, Association of Directors of Anatomic and Surgical Pathology and the World Health Organization for reporting carcinoma extent have been summarized. Given these recommendations, the extent parameters currently in use in AS protocols and the evidence from the literature, it is suggested that pathologists should report the absolute number of involved cores out of total number cores and the amount of cancer in the single core with the greatest amount of tumor expressed as the percentage involvement by carcinoma, with or without the linear extent of carcinoma in that core. Percentage involvement by carcinoma and or linear extent of carcinoma in each positive core may also be provided. All other measurements are optional. Linear extent of carcinoma in each core may also be provided. Other measurements are optional.

The extent of cancer in prostate needle biopsy cores in patients potentially candidates for AS should based on the recording of:

1. Number of positive cores/total number of cores. The number of positive cores could possibly affect subsequent therapy in terms of suitability for AS, such that it is justified to perform an immunohistochemical work-up of additional atypical foci (either HMWCK or p63 or combination of the two with AMACR).

2. Linear percentage of prostatic tissue involved and/or total linear measurement of carcinoma and total core length. This can be done calculating the percentage of each core involved by cancer, based on the linear length of cancer $(\mathrm{mm})$ divided by the core length, then multiplied by 100 . The other method is to provide a percentage estimate of involvement of each of the cores derived by visual estimation. It has been shown that the former is more accurate and reproducible when patients are evaluated for eligibility for an AS protocol.

\section{Problems associated WITH TUMOR EXTENT MEASUREMENTS}

\section{Measuring discontinuous foci of cancer}

When measuring discontinuous foci of cancer on a prostate needle biopsy core, the pathologist has to specify presence of discontinuous foci, linear extent in aggregate of discontinuous foci, percentage involvement of the core, and the core length spanned by discontinuous foci. The following sentence is suggested as a template for the pathology report: "Prostate biopsy core (Length: $1.2 \mathrm{~cm}$ ) with two discontinuous foci, measuring 1.5 and $1.2 \mathrm{~mm}$ (measuring $2.7 \mathrm{~mm}$ in aggregate), respectively, of Gleason score $3+3=6$ acinar PCa separated by $4 \mathrm{~mm}$ of intervening benign tissue. The tumor spans 56\% of the core length, involving $22 \%$ of the core".

\section{Tissue core and tumor fragmentation}

Concerning the number of cores per cassette, the ideal would one core per cassette. Two biopsies from the same location could be embedded together. It has been shown that simultaneous inclusion of 3 biopsies in the same cassette can lead to the loss of a mean length of $1.15 \mathrm{~cm}$ of assessable tissue which corresponds to the average length of one prostate biopsy.

When multiple cores are submitted in a single cassette or jar by the urologist and processed in a single cassette, many pathologists give the overall percentage of cancer for the entire slide as opposed to the percentage for each individual core. At the Pathology Laboratory of United Hospitals, Ancona, we attempt to give the percentage of cancer per core for each individual positive core, regardless of how many cores are on a given slide. 
When reporting biopsies with multiple cores in the same jar, the pathologist has to provide linear measurement (in millimeters) or linear extent (as percentage) of prostatic tissue involved for the most involved core and the overall linear percentage or measurement in millimeters of tissue submitted from the site(s) with cancer.

If there are multiple fragmented small cores containing cancer, an accurate assessment of percentage of cancer per core cannot be determined, and only an overall percentage of cancer per fragmented specimen can be noted. In this scenario, one cannot even determine with certainty the number of positive cores. There is evidence in the literature that there is a greater tendency to core fragmentation when $>1$ core is submitted in a container. It is our experience that needle biopsies collected onto gauze or paper are more likely to fragment. Assessment of number of cores involved by $\mathrm{PCa}$ is difficult in the presence of core fragmentation. A comment should be made suggesting that the urologist/clinician obtaining the biopsy is in the best position to make determination of number of cores involved based on the original submission of number of cores, and, if necessary, to undertake clinico-pathologic correlation (19).

\section{Minimum acceptable core length}

Currently there is no definition for adequate or minimum acceptable core length. The percentage of cancer in a short core (e.g. $<5-10 \mathrm{~mm}$ ) versus that in a sufficiently long core mean entirely different tumor lengths. This has implications for interpretation of percent core involvement in the setting of AS. Since percent core involvement is based only on total length of prostatic parenchyma, non-prostatic elements should not be included in total core length assessment.

\section{GLEASON SCORE}

The previous decade has seen considerable change in practice relating to Gleason grading of prostatic carcino$\mathrm{ma}$, and in 2005 the International Society of Urological Pathology (ISUP) undertook a major revision of the Gleason grading system. This was designed to reflect current practice and to incorporate recently gained knowledge on the biology of prostate cancer. The ISUP 2005 modification of the Gleason Grading System has resulted in changes to the definitions of Gleason patterns 3 and 4 tumors. This is of particular importance for those patients in which deferred treatment is contemplated, as grade is central to the criteria utilized for identifying patients suitable for inclusion in AS programs. Two features define Gleason pattern 3 glands: clearly infiltrating glands (in contrast to the overall nodular configuration of glands in Gleason patterns 1 and 2) and each gland being a single discrete individual glandular structure that is well formed. The 2005 ISUP Modification of the Gleason grading system defined virtually all cribriform glands as Gleason pattern 4, although in this classification well circumscribed, small, ovoid to round cribriform glands with regular bridging were included in Gleason pattern 3. More recently it has been suggested that as all cribriform glands appear to be associated with a less favorable prognosis; these glands should also be classified as pattern 4. Glands with a glomeruloid architecture are also considered as pattern 4. Using these criteria, classification as Gleason pattern 3 should be confined to tumors consisting of well formed, separate glands with lumina.

Accurate distinction of Gleason pattern 3 from Gleason pattern 4 is critical for eligibility for most AS protocols. When there is doubt, it is suggested:

- Defaulting to a lower grade,

- Following the focus on deeper serial sections,

- Sharing such borderline cases with a colleague as the presence of a Gleason pattern 4 may preclude the patient from AS. Biopsy Gleason grade reclassification has emerged as one of the most meaningful intervention criteria for men on AS.

\section{Situations that SHOULD EXCLUDE a PATIENT FROM AS}

The presence of perineural invasion does not represent an exclusion criterion. However, there are rare pathologic situations that should likely exclude a patient from AS:

- Histologic types: prostatic adenocarcinoma with predominant ductal carcinoma histology, sarcomatoid carcinoma, small cell carcinoma

- Intraductal carcinoma without invasive carcinoma

- Extraprostatic extension in needle biopsy

- Lymphovascular invasion in needle biopsy.

\section{Conclusions}

Given the overdiagnosis and overtreatment of low risk prostate cancer, AS should be a ubiquitously adopted and formalized strategy. Men who experience early upgrading likely represent initial sampling error, whereas later upgrading may reflect tumor dedifferentiation. There are several issues and key questions that arise from the combined clinico-pathologic experience from formal and informal AS treatment management strategies that would be applicable to the management of prostate cancer patients outside of academic centers and clinical trials. These need to be resolved over the next few years to tighten criteria of selection for patients contemplating AS and their subsequent management.

\section{REFERENCES}

1. Heidenreich A, Bastian PJ, Bellmunt J, et al. EAU guidelines on prostate cancer. part 1: screening, diagnosis, and local treatment with curative intent-update 2013. Eur Urol. 2014; 65:124-37.

2. Carter HB, Kettermann A, Warlick C, et al. Expectant management of prostate cancer with curative intent: an update of the Johns Hopkins experience. J Urol. 2007; 178:2359-64.

3. Dall'Era MA, Konety BR, Cowan JE, et al. Active surveillance for the management of prostate cancer in a contemporary cohort. Cancer. 2008; 112:2664-70.

4. Ercole B, Marietti SR, Fine J, Albertsen PC. Outcomes following active surveillance of men with localized prostate cancer diagnosed in the prostate specific antigen era. J Urol. 2008; 180:1336-9.

5. Roemeling S, Roobol MJ, de Vries SH, et al. Active surveillance for prostate cancers detected in three subsequent rounds of a screening trial: characteristics, PSA doubling times, and outcome. Eur Urol. 2007; 51:1244-51 
6. Van As NJ, Norman AR, Thomas K, et al. Predicting the probability of deferred radical treatment for localised prostate cancer managed by active surveillance. Eur Urol. 2008; 54:1297-305.

7. Dall'Era MA, Cooperberg MR, Chan JM, et al. Active surveillance for early-stage prostate cancer: review of the current literature. Cancer. 2008; 112:1650-9.

8. Van den Bergh RCN, Roemeling S, Roobol MJ, et al. Prospective validation of active surveillance in prostate cancer: the PRIAS study. Eur Urol. 2007; 52:1560-3.

9. Klotz L. Active surveillance with selective delayed intervention is the way to manage "good-risk" prostate cancer. Nat Clin Pract Urol. $2005 ; 2: 136-42$.

10. Lacetera V, Galosi AB, Cantoro D, et al. Transrectal ultrasound (TRUS) and TRUS-biopsy's accuracy in potential candidates for PRIAS active surveillance protocol but treated with immediate radical prostatectomy. Arch Ital Urol Androl. 2012; 84:4:272-275.

11. Ploussard G, Xylinas E, Salomon L, et al. The role of biopsy core number in selecting prostate cancer patients for active surveillance. Eur Urol. 2009; 56:891-8.

12. Quintal MM, Meirelles LR, Freitas LL, et al. Various morphometric measurements of cancer extent on needle prostatic biopsies: which ispredictive of pathologic stage and biochemical recurrence following radicalprostatectomy? Int Urol Nephrol. 2011; 43:697-705.

13. Bismar TA, Lewis JS Jr, Vollmer RT, Humphrey PA. Multiple measures of carcinoma extent versus perineural invasion in prostate needle biopsy tissue in prediction of pathologic stage in a screening population. Am J Surg Pathol. 2003; 27:432-40.

14. Park HJ, Ha YS, Park SY, et al. Incidence of upgrading and upstaging in patients with low-volume Gleason score 3+4 prostate cancers at biopsy: finding a new group eligible for active surveillance. Urol Int. 2013; 90:301-5.

15. Brimo F, Montironi R, Egevad L, et al. Contemporary grading for prostate cancer: implications for patient care. Eur Urol. 2013; 63:892-901.

16. Poulos CK, Daggy JK, Cheng L. Prostate needle biopsies: multiple variables arepredictive of final tumor volume in radical prostatectomy specimens. Cancer. 2004; 101:527-32.

17. Lewis JS Jr, Vollmer RT, Humphrey PA. Carcinoma extent in prostate needle biopsy tissue in the prediction of whole gland tumor volume in a screening population. Am J Clin Pathol. 2002; 118:442-50.

18. Rubin MA, Bismar TA, Curtis S, Montie JE. Prostate needle biopsy reporting: how are the surgical members of the Society of Urologic Oncology using pathology reports to guide treatment of prostate cancer patients? Am J Surg Pathol. 2004; 28:946-52.

19. Galosi AB, Muzzonigro G, Lacetera V, Mazzucchelli R. Specimen orientation by marking the distal end: (potential) clinical advantages in prostate biopsy. Prostate Cancer. 2011; 2011:270403.

\section{Correspondence}

Roberta Mazzucchelli, MD

r.mazzucchelli@univpm.it

Marina Scarpelli, MD

m.scarpelli@unvpm.it

Rodolfo Montironi, MD (Corresponding Author)

r.montironi@univpm.it

Pathological Anatomy, Polytechnic University of the Marche Region,

School of Medicine, United Hospitals,

Via Conca 71, I-60126 Torrette, Ancona, Italy

Andrea Benedetto Galosi, MD

galosiab@yahoo.it

Division of Urology, "Augusto Murri" General Hospital, ASUR Marche Fermo, Italy

Antonio Lopez-Beltran, MD

emllobea@gmail.com

Department of Surgery, Cordoba University Medical School

Cordoba, Spain

Liang Cheng, MD

linag_cheng@yahoo.com

Department of Pathology and Laboratory Medicine,

Indiana University School of Medicine, Indianapolis, IN, USA 